\title{
Research on Crucial Techniques of Smart Distribution Grid
}

\author{
Zhigang $\mathrm{Wu}^{1}$, Wei Wang ${ }^{1}$, Jianqiang $\mathrm{Xu}^{1}$, Yuqiang $\mathrm{Xu}^{1}$, Jidong Shi ${ }^{2}$, Dongju Wang ${ }^{2}$ \\ ${ }^{1}$ State Grid Zhejiang Tongxiang Power Supply Company \\ Tongxiang, 314500, China \\ ${ }^{2}$ College of Electrical Engineering, Zhejiang University \\ Hangzhou 310027, China \\ Correspondence should be addressed to Shi Jidong; jidong_shi@163.com
}

\begin{abstract}
Smart distribution grid is the important part of smart grid which connects the main network with users. It is directly user oriented and the center of power distribution. This article has compared the smart distribution grid with regular traditional distribution network. Crucial techniques including automation technology, Informationization technology and interaction are the emphasis of discussion. The results have indicated that researches on advanced electric equipment, online monitoring, self healing, advanced metering infrastructure, integrated communication, micro gird and other technologies would be the principal directions of study on smart distribution grid, simultaneously providing reference for realization of practical application in future.
\end{abstract}

Keywords- Smart distribution grid; Automation technology; Informationization technology; Interaction technology

\section{INTRODUCTION}

The development of low carbon, green and circular economy indicates that the future operation mode of power network should be economic, efficient, reliable and safe. The utilization of renewable energy in large scale is an important means to maximize economical and social benefits of available energy. These new developing directions belong to the newly proposed concept of smart grid, which has become the focus of social attention

As an important part of smart grid, the smart distribution grid is directly user oriented and having decisive effects on electric power quality. Its power loss, investment and operation cost greatly exceeds those of the transmission network. Nonetheless, the actual investment in distribution network in out country is obviously not enough. The automation and intellectualization level has fallen behind and become the bottleneck of power system development. Besides, the integration of distributed generation also brings new challenges to the operation and control method of distribution network ${ }^{[1-3]}$. After years of exploration, experts all over the world have obtained research results and gained much practical experience of smart distribution grid technology. Based on current research situation, this article has illustrated characteristics and advantages of smart distribution grid. Automation, informationization and interaction technology stand for the future development trend.

\section{CHARACTERISTICS AND ADVANTAGES OF SMART DISTRIBUTION GRID}

Compared with traditional distribution network, smart distribution grid possesses a series of characteristics and advantages.

Firstly, power supply would be more reliable. With the aid of real-time fault detection device, the control center could quickly respond to faults and minimize affection on users.

Secondly, electric power quality would be better. Different kinds of power electronic facilities can be used to optimize control of voltage and reactive power and improve electric power quality. For example, the dynamic voltage compensator is able to guarantee continuous power supply during the process of fault treatment and reclosing. The solid state circuit breaker is used to realize seamless switch of power sources and continuous power supply of important power consumer equipment.

Thirdly, the massive integration of distributed generation is applicable. Distributed generation including wind power, solar energy and biomass energy can be effectively integrated with the distribution network, realizing energy conservation and emission reduction meanwhile improving operation efficiency of the network. Besides, when blackout accident happens in the main network the micro-grid with distributed generation can support power supply of important users.

Fourthly, power management can be carried out by users. The smart distribution grid supports the function of smart meter and intercommunication with users. The real-time electricity price provides opportunities for users to establish their own power consumption plans, which is good for peak load cutting. Plus, users can have self-supply power generation service and power storage facilities.

Fifthly, the operation state of the network and facilities can be monitored and adjusted. By real-time data recollection the system can provide real-time information like power flow, load distribution, states of facilities, electric power quality, fault position and fault area. Based on these the system can control power flow, minimize power loss, reduce capacity-load ratio and raise the load rate.

At last, the resource utilization rate is higher. The operation status of important equipment is monitored at all time. The condition based maintenance is of great help 
extending service lives of facilities. On the other hand, the system also supports fast simulation of distribution system and control of power flow. By which higher capacity-load ratio is derived and representing higher utilization rate of system capacity. With lower investment and equipment depreciation speed the electricity price can be decreased without sacrificing electric power quality.

\section{CRUCIAL TECHNOLOGY OF SMART DISTRIBUTION GRID}

\section{A. Automation Technology}

The automation technology applied in distribution network includes automatic selection of control strategy, real-time monitoring of operation state and fault recovery, also known as feeder automation and distribution management system. To guarantee highly efficient quality of the system advanced electric equipment, online monitoring, self-healing and other techniques are of great importance $^{[4-6]}$

(1)Advanced electric equipment technology. The intelligent primary equipment technology contains intelligent circuit breaker, superconductor cable, composite wire, advanced power storage facilities, electronic equipment, optical transducer and other auxiliary equipment. The application of microprocessor and optoelectronic technology in signal detection and controlling driving devices has tremendously simplified the structure of regular electro mechanical relay and its control circuit. The traditional connecting wire has also been replaced by digital signal control.

The intelligent secondary equipment technology aims at digitalization and network of secondary equipment. By using microprocessors based on standard module to design and manufacture secondary electric equipment, the connection between facilities has adopted the high speed network communication technology. Communication protocol and optical fiber could improve the efficiency of data and source sharing. The transmission of data could be standardized, objective and more reliable.

(2)Online monitoring and condition based maintenance technology. This technology is concentrated on state monitoring, fault detection and treatment. Depending on continuous monitoring, service predication and reliability estimation of electric equipment, the working staff could pertinently establish maintenance schedule. Other than blind periodical inspection, this technology is more effective and economic. The infrared diagnostic technology, distant diagnostic technology and distributed monitoring and diagnosis system could make it possible to obtain operating state of any equipment at any time and overcome obvious shortages of regular preventive tests. Above all, the development of online monitoring has accelerated the intellectualization of network management. Fast and convenient data exchange remarkably shortens the reaction time when fault happens and reduces outage cost.

(3)Self-healing technology. The concept of self-healing firstly appears in the complex interaction system research project launched by electric power research institute (EPRI) and U.S. Department of Energy in 1999. Afterwards the projects of smart gird and modern gird initiative have regarded self-healing technology as the core technical means of improving electric power quality and highlighted research direction of smart grid.

The self-healing technology in distribution network means using continuous online self estimation and automatic preventive operation to quickly find out and eliminate potential faults. When fault happens the system should have its own countermeasures and minimize fault area. This technology mainly consists of probabilistic risk assessment and preventive control and power overload avoidance through load prediction and control strategy adjustment in advance. Protective relays and automation equipments should eliminate faults or at least isolate fault area to avoid large-scale blackout.

(4)Advanced Metering Infrastructure (AMI) technology. The AMI is the higher level of automatic metering reading (AMR). The research of advanced metering and communication technology is the basis of developing smart distribution grid. Typical AMI system is comprised of intelligent ammeter, intercommunication network, metering data management system (MDMS) and home area network (HAN).

The intelligent ammeter is the basic data collector in distribution network. It could periodically check power consumption, voltage and current.

The intercommunication network is responsible for transmitting data from smart ammeters to data center. The uploaded data could support different functions of the distribution information system. On the other hand, real-time electricity price and other information like demand response could be delivered to users through intercommunication network. The timely exchange of information could realize interaction between distribution system and users.

The basic function of MDMS is to confirm, edit and evaluate data from AMI. Even when communication network fails or fault happens at user side, the MDMS could still ensure the integrity and accuracy of the uploaded data stream

The home area network means connection with controllable electrical apparatus inside the house of user. By this means the household appliances directly connect with smart ammeter through network gateway. The electricity price and power consumption would be accessible to users at all time. According to power market requirement or system demand, the load distribution can be adjusted. Typical application of this technology is that some equipment could be automatically turned on when system capacity is sufficient, otherwise turned off. So users can be 
aware of power consumption and market situation timely and accurately, which is beneficial for healthy operation of the network and energy saving.

\section{B. Informaionization Technology}

(1) Integrated communication technology. The communication technology includes integrated communication system, establishing and testing of communication protocol, wireless communication and virtual private network techniques. Currently, common communication methods are broadband over power lines (BPL), optical fiber, wired and wireless techniques like GPRS and 3G. The latest BPL technology is based on digital signal process chip. Its powerful function of real-time decoding makes it an ideal communication method. Optical fiber is more and more concerned because of its outstanding characteristics of safety, easy realization and strong anti-interference capability. However, the optimal communication method should be union of several techniques. The final destination is to establish high speed and bi-directional communication system. With information integration and massive data calculation techniques, the smart sensors, control system, protection system, power supply management system of smart distribution network could dynamically interact with users and realize data exchange $^{[7-9]}$.

(2) Information integration of power management. Information stream is control center and neural system of distribution network. The automation and Informationization of real-time monitoring and management needs to share and integrate different kinds of information from all sides. The information transmission of smart distribution network should obey the same rule. Public communication protocol and data transmission channel would be the basis of information integration. Data uploaded from different facilities needs a platform to be transformed and exchanged. Presently, the mainstream of information integration architecture could be categorized as integrated architecture based on traditional data warehouse, technical architecture based on CORBA/DCOM and integrated architecture based on web service. The whole system usually consists of data source layer, information integration platform, information application layer, and information portal layer. The high integration level of real-time and off line distribution network data makes the Informationization of equipment maintenance and power consumption management come true.

\section{Interaction Technology}

(1)Micro grid Technology. The micro grid (MG) is a small sized power system containing distributed generation, power storage equipment, power transformation facilities, load monitoring and protection devices. It can connect with

\footnotetext{
*Special description of the title. (dispensable)
}

the main network or control, manage and operate totally on its own. As an important part of smart grid, the micro grid has advanced technology in measurement and control, protection, power electronics. It can flexibly establish or cut loose the connection with main network. When outrage happens in main network, the micro grid can provide power supply for important equipment. Making full use of micro grid could improve the self healing ability and power supply reliability.

When micro grid is used to transfer power to main network and join electric market. It decides the detailed interaction method by considering local electric power and fuel consumption, demand of electric power quality, electricity price, service requirement, demand side management and other problems. The real-time frequency regulation, power balance, voltage stabilization and black start are symbols of the high intellectualization level of micro gird technology. When micro grid is isolated from main network, it operates as an controllable power load. Its own power factor and load distribution would be within grasp, which guarantees the stability of system operation.

(2)Interaction with users. The interaction of smart distribution grid concerns power resources, power network and users. It has two aspects of interaction of power consumption information and demand side response. The former applies modern communication technology in realizing real-time data exchange between power generation enterprises and users. One example is the data collection function of smart ammeter. The users accept information about outrage arrangement and other power consumption news. The demand side response means supporting integration of massive distributed renewable energy power generation and power demand of electric vehicle. Through interaction with users the electric market could operate in better condition. The enthusiasm for users to take part in network management could then be ignited.

(3)Decision support and human-computer interaction. The decision support function provides data, model and knowledge for decision makers to analyze problems, build models and simulate judging process. With different information sources and analysis tools the decision making level and quality could be optimized. The human-computer interaction technology is based on artificial intelligence and containing advanced operation interfaces and decision techniques like visualization, humanistic human machine interface, voice interaction terminal, multi-functioned software system, performance instrument panel and real-time dynamic simulation. The dispatchers, operators, maintainers and other managers could keep in touch and communicate with each other conveniently and effectively.

\section{CONCLUSIONS}

The development of smart distribution grid is an important part of smart grid. It is of great use coping with 
future challenges of resource shortage, environmental deterioration, economic and social difficulties. Other problems like weak structure of distribution network, low automation level, negative affection brought by massive integration of distributed generation, limited interaction and communication between power generation enterprises and users can also be effectively dealt with if the smart distribution technology becomes mature.

This article has systematically introduced characteristics and advantages of smart distribution grid. One step further, crucial techniques including automation technology, informationizaiton technology and interaction technology were detailed and analyzed, which provides reference for further research directions of smart distribution grid.

\section{ACKNOWLEDGEMENT}

On the completion of my thesis, I should like to express my deepest gratitude to all those whose kindness and advice have made this work possible. I am greatly indebted to Wang Wei who gave me valuable instructions and has improved me in language. His effective advice, shrewd comments have kept the thesis in the right direction. I would like to thank my partner $\mathrm{Xu}$ Jianqiang and $\mathrm{Xu}$ Yuqiang for their friendship and constructive suggestions; they constantly encouraged me when I felt frustrated with this dissertation.

\section{REFERENCES}

[1] Li Tianyou and Xu Bingyin. "Self-healing and its benchmarking of smart distribution grid”. Power System Protection and Control, vol.
38, no. 22, pp. 105-108, 2010.

[2] Wang Chengshan, Wang Shouxiang and Guo Li. "Prospect over the techniques of smart distribution network in China". Southern Power System Technology, vol. 4, no. 1, pp. 18-22, 2010.

[3] Li Xun, Gong Qingwu, Hu Yuanchao, Yang Qunying and Liang Wenteng. "Discussion of smart distribution grid system". Electric Power Automation Equipment, vol. 31, no. 8, pp. 108-111, 2011.

[4] Gu Xinxin, Jiang Ning, Ji kan, et al. "Practice and Prospects of self-healing control of intelligent distribution grid”. Electric Power Construction, vol. 30, no. 7, pp. 4-6, 2009.

[5] Xu Bingyin, Li Tianyou and Xue Yongduan. "Smart distribution grid and distribution automation". Automation of Electric Power Systems, vol. 33, no. 17, pp. 38-42, 2009.

[6] Luan Wenpeng. "Advanced metering infrastructure". Southern Power System Technology, vol. 3, no. 2, pp. 6-10, 2009.

[7] Han Guozheng, Xu Bingyin and Zhang Haitai. "Application of IEC61850 in advanced distribution automation". Electric Power Automation Equipment, vol. 31, no. 5, pp. 99-102, 2011.

[8] Li Xiangzhen, He Qingsu and Sun Jisheng. "Research and application on communication technology in smart distribution and utilization grid”. Electric Power, vol. 44, no. 12, pp. 78-81, 2011.

[9] Zhu Ruijie, Yu Fang and Xia Zengji. "Application of EPON in power distribution automation”. Electric Power Construction, vol. 32, no. 6, pp. 49-53, 2011.

[10]Zhao Hongwei and Wu Taotao. "Review of distributed generation based microgrid technology”. Proceedings of the CSU-EPSA, vol. 20, no. 1, pp. 121-128, 2008.

[11]Wang Chengshan and Li Peng. "Development and challenges of distributed generation, the micro-grid and smart distribution system". Automation of Electric Power Systems, vol. 34, no. 2, pp. 10-14, 2010. 